\title{
Successful monozygotic triplet pregnancy after a single blastocyst transfer following in vitro maturation of oocytes from a woman with polycystic ovary syndrome: a case report
}

\author{
Kuniaki Ota ${ }^{1}$, Toshifumi Takahashi ${ }^{1 *}$ (D), Mikiko Katagiri² ${ }^{2}$ Ryu Matsuoka ${ }^{3}$, Akihiko Sekizawa ${ }^{3}$, Hideki Mizunuma ${ }^{1}$ and \\ Hiroaki Yoshida ${ }^{2}$
}

\begin{abstract}
Background: Although women with polycystic ovarian syndrome (PCOS)-related sub-fertility are high responders to controlled ovarian stimulation, it is difficult to obtain mature oocytes in these women. Therefore, in vitro maturation (IVM), which is the technique of letting the contents of the ovarian follicles and the oocytes inside mature in vitro, has often been proposed in such women. We describe the first successful delivery of monozygotic triplets resulting from transfer of a single blastocyst following IVM of oocytes.
\end{abstract}

Case presentation: A 32-year-old nulligravida female with PCOS underwent IVM. She underwent vitrifiedwarmed single blastocyst transfer following IVM, and a dichorionic triamniotic triplet pregnancy was confirmed at 8 weeks. Healthy triplets were delivered by cesarean section at 33 weeks' gestation. This is the first case of monozygotic triplets derived from IVM oocytes that were successfully delivered. The determination of chorionicity and amnionicity is generally supposed until 3 days after fertilization, and no division or splitting of her embryo was observed on transfer. Interestingly, her embryo might have split after the transfer, resulting in a dichorionic triamniotic triplet pregnancy.

Conclusions: Patients should be informed of a possible increased risk of monozygotic multiple pregnancies after single embryo transfer following IVM.

Keywords: Assisted reproductive technology, In vitro maturation of oocyte, monozygotic triplets, polycystic ovary syndrome, Zygotic splitting

\section{Background}

However, even if the single embryo transfer (SET) is performed, the multiple pregnancies, such as twin or more pregnancies, has been reported [1-3]. So far, there is evidence that the rate of monozygotic multiple pregnancies by assisted reproductive technology (ART)

\footnotetext{
* Correspondence: totakaha@fmu.ac.jp

${ }^{1}$ Fukushima Medical Center for Children and Women, Fukushima Medical University, 1 Hikarigaoka, Fukushima City, Fukushima 960-1295, Japan Full list of author information is available at the end of the article
}

$(1.01-2.24 \%)$ is higher than those by spontaneous conception (0.40-0.45\%) [4-7]. Although the precise mechanisms of higher rate of monozygotic splitting by ART treatment remain unknown, there have been reported about risk factors for monozygotic splitting, such as zona or oocyte membrane breaking for intracytoplasmic sperm injection (ICSI), embryo biopsy, and assisted hatching, and extended culture period for blastocyst-stage embryo culture [1-3, 8, 9].

In vitro maturation (IVM) of immature oocytes is a promising method for collecting eggs without side 
effects, such as ovarian hyperstimulation syndrome (OHSS), in polycystic ovary syndrome (PCOS) women [10]. Many cases of pregnancy and delivery of healthy children by ART treatments using oocytes derived from IVM of immature oocytes in PCOS women [11]. To date, the combined technique of cryopreservation and IVM is established treatment to prevent OHSS in PCOS women $[12,13]$ and this strategy obtains a pregnancy rate of up to $35-40 \%$ [14]. However, this method assumes various unclear risks associated with long-term culture.

There have been some reports that SET following IVM of immature oocytes retrieved from infertile woman resulted in twin pregnancy $[15,16]$, but it has not been clarified in IVM whether a longer culture time may cause zygotic splitting. Nevertheless, the actual number of births from IVM is not clear because IVM is performed in only a limited number of institutes worldwide. Herein, we describe our first experience with the first successful delivery of monozygotic triplets resulting from single blastocyst transfer following IVM of oocytes.

\section{Case presentation}

A 32-year-old nulligravida Asian female visited the Sendai Art Clinic with primary infertility, including anovulation for the past 2 years. She was diagnosed with PCOS according to the Rotterdam ESHRE/ ASRM consensus criteria [17]. Hysterosalpingography showed bilateral tubal patency and no defects of uterine cavity. Her husband's semen analysis showed normozoospermia, based on the 2010 World Health Organization criteria. After she had failed to achieve pregnancy following several courses of ovulation induction with clomiphene citrate or gonadotrophin injections, she opted for IVM treatment to retrieve germinal vesicle (GV) oocytes for in vitro culture. Informed consent was provided.

She was initiated on an IVM-in vitro fertilization (IVF) protocol on day 6 of a spontaneous menstrual cycle and given 4 days of $75 \mathrm{IU}$ recombinant FSH (Gonal-F, Serono Japan, Tokyo, Japan). She was repetitively evaluated on days 6 and 8 of the menstrual cycle by transvaginal ultrasound scans. When the leading follicle size was less than $12 \mathrm{~mm}, 5000 \mathrm{IU}$ of urinary human chorionic gonadotropin (hCG) (gonatropin, ASKA Pharmaceutical Co., Ltd., Tokyo, Japan) was administered and oocyte retrieval was carried out $34 \mathrm{~h}$ after hCG triggering. The collection and culture of immature oocytes were performed as previously described [15].

Fifteen GV stage immature oocytes were retrieved and incubated for $26 \mathrm{~h}$ in IVM culture medium (MediCult IVM medium, ORIGIO Japan, Yokohama, Japan) supplemented with recombinant FSH $75 \mathrm{mIU} / \mathrm{ml}$ (Gonal-F, Serono Japan, Tokyo, Japan), hCG $100 \mathrm{mIU} / \mathrm{ml}$ (gonatropin, ASKA Pharmaceutical Co., Ltd., Tokyo, Japan), and $10 \%$ patient's inactivated serum. The oocytes were then denuded from the cumulus cells. Eleven mature oocytes were obtained and subjected to ICSI with fresh sperm (Fig. 1). Zygotes were cultured in culture medium (Universal IVF, ORIGIO Japan, Yokohama, Japan), and fertilization was judged 16-18 h after ICSI for the appearance of two distinct pronuclei and two polar bodies. Nine (81.8\%) oocytes were fertilized. Fertilized zygotes were transferred into culture medium (IVC-1, In Vitro Care, MD, Frederick, USA) and all embryos developed to blastocysts on day 5 (one middle expanding blastocyst and early blastocysts) and were cryopreserved by vitrification.

For the preparation of the endometrium, transcutaneous estradiol (Estrana TAPE $0.72 \mathrm{mg}$, Hisamitsu Pharmaceutical, Tokyo, Japan; four sheets every 2 days) was administered on day of the hormone replacement therapy (HRT). The endometrial lining was evaluated on days $12-14$ of the HRT cycle and the endometrial thickness needed to be a minimum of $8 \mathrm{~mm}$. A vaginal progesterone suppository (Luteum, Aska pharmacy, Tokyo, Japan; 2 tablets everyday) was administered when the endometrial thickness achieved was greater than $8 \mathrm{~mm}$. The transcutaneous estradiol and vaginal progesterone suppository were continued until 9 weeks of gestation. A single frozen-thawed blastocyst (Gardner's classification:4 BC) (Fig. 2) was transferred under transvaginal ultrasound guidance on day 5 after vaginal progesterone suppository was administered. Two weeks

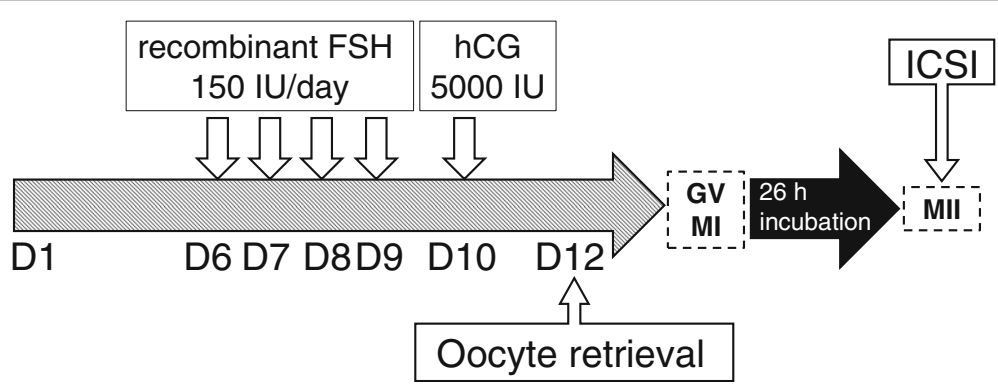

Fig. 1 Time course of the invitro maturation-in vitro fertilization protocol. FSH: follicle stimulating hormone, hCG: human chorionic gonadotropin, GV: germinal vesicle, MI: metaphase I, MII: metaphase II, ICSI: intracytoplasmic sperm injection 


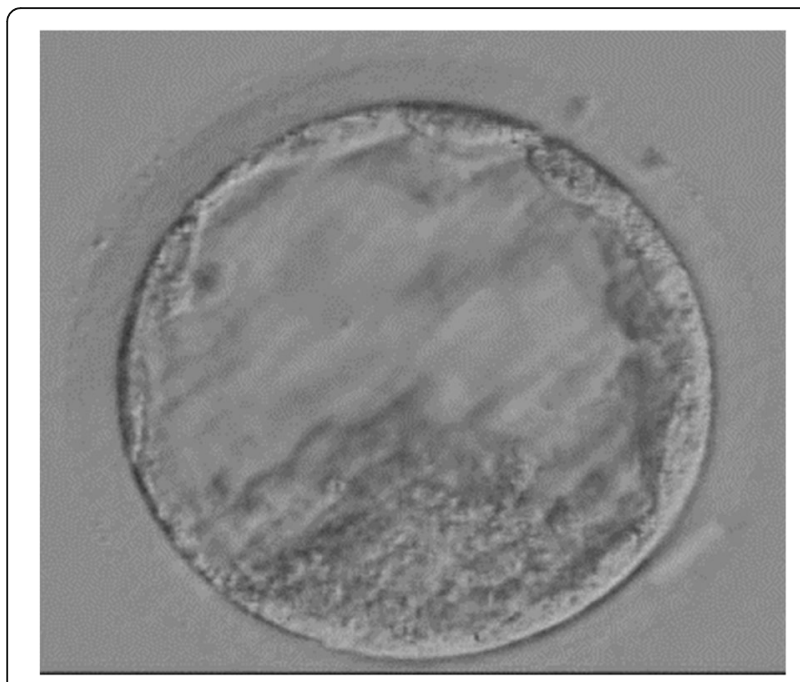

Fig. 2 Vitrified-warmed blastocyst graded to 4 BC according to Gardener's classification

after blastocyst transfer, the patient's serum hCG concentration was $2194 \mathrm{IU} / \mathrm{L}$ and pregnancy was confirmed. Transvaginal ultrasound performed at 5 weeks and 0 days of gestation showed 2 gestational sacs inside the uterus. At 8 weeks 2 days gestation, 3 fetal heart beats were detected and a dichorionic triamniotic triplet pregnancy was observed by ultrasound (Fig. 3). The subsequent pregnancy course went well until 28 weeks 5 days when she was admitted to the maternal fetus intensive care unit for fetal growth restriction. She received prenatal steroids for fetal lung maturation and intravenous magnesium sulfate for tocolysis until the delivery. At 33 weeks 4 days, three baby girls were delivered by an elective Caesarean section. Their weight and APGAR scores at $1 \mathrm{~min}$ and $5 \mathrm{~min}$ were $1852 \mathrm{~g}$, 8-8, 1817 g, 7-8, and 1495 g, 8-9, respectively. Histological examination of the placenta confirmed monochorionicity. The babies remained in the neonatology unit without morbidity and were discharged 4 weeks after birth.

\section{Discussion and conclusions}

Here, we describe a case of a triplet pregnancy from a SET following IVM with a successful delivery at 33 weeks. This triplet pregnancy was considered dichorionic derived from monozygotic embryos following IVM. This patient was diagnosed with anovulatory PCOS, and therefore, a concurrent spontaneous pregnancy at the same time as SET was not considered. Therefore, this pregnancy was considered monozygotic triplets and, moreover, the gender was the same female. To the best of our knowledge, this is the first report on a

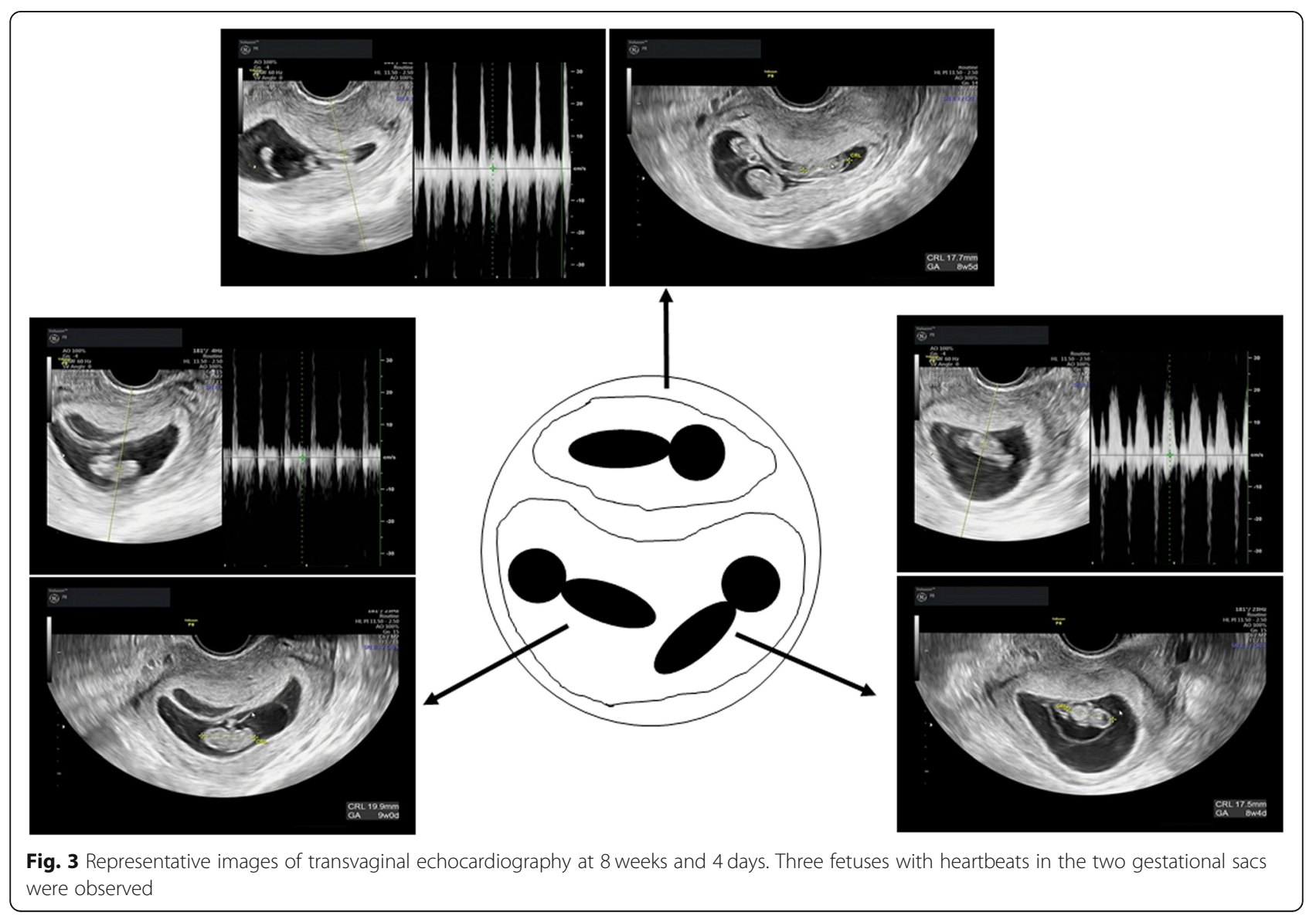


successful delivery of monozygotic triplets after SET following IVM.

A dichorionic twins occurs when an embryo splits until 3 days after fertilization and a monochorionic twins occurs when an embryo splits 4-8 days after fertilization [18]. These phenomena are speculated based on natural pregnancy. In the ART treatment cycles, dichorionic twins originated from SET have been reported $[19,20]$. This does not fit the theory of embryo splitting timing and forming monozygotic dichorionic twins. In the present case, none of the embryo split was observed at the time of transfer 5 days after fertilization. There is a possibility that the embryo split after the transfer and became a dichorionic triamniotic triplet.

Very recently, Ikemoto et al. reported that the prevalence of a triplet pregnancy after SET following IVF and ICSI was $0.04 \%$ in the Japanese ART data [21]. However, their data cannot distinguish a triplet pregnancy following IVM. On the other hand, due to the small number of babies born following IVM worldwide [22, 23], it is not possible to mention the relationship between IVM and monozygotic triplets.

There is currently little data on the mechanisms of zygotic splitting leading to monozygotic multiple pregnancies. Several factors have been discussed in the literature but not easy to take off form the hypothesis. Generally, possible risk factors for the increasing rate of zygotic splitting has been speculated prolonged culturing of embryos and zona pellucida manipulation, such as assisted hatching, ICSI, and embryo biopsy [24-28]. IVM may increase the occurrence of zygote splitting due to longer in vitro culturing before insemination. In fact, in the present case, embryos were cultured in vitro for 6 days ( 1 day before fertilization and 5 days after ICSI). Therefore, the extension of the culture time was one of the most speculated cause for zygotic splitting. However, at present, the cause of zygotic splitting remains unknown. The patient's genetic predisposition, including PCOS itself, the effects of drugs administered before oocytes collection, and the effects of in vitro maturation culture may also be related to zygotic splitting [29].

In conclusion, we report here the first case of a monozygotic triplet pregnancy from IVM oocytes with a successful delivery after intensive perinatal care. Patients should be informed of a possible increased risk of monozygotic multiple pregnancies after SET. The frequency and mechanism of how the monozygotic triplet pregnancy occurs during the IVM is still unknown. Future studies should be needed to clarify the relationship between zygotic splitting and IVM and other ART procedures.

\section{Abbreviations}

ART: Assisted reproductive technology; ET: Embryo transfer; GV: Germinal vesicle; hCG: human chorionic gonadotropin; HRT: Hormone replacement therapy; ICSI: Intracytoplasmic sperm injection; IVF: In vitro fertilization; IVM: In vitro maturation; OHSS: Ovarian hyperstimulation syndrome; PCOS: Polycystic ovary syndrome; SET: Single embryo transfer

\section{Acknowledgements}

We gratefully acknowledge the contributions of the other members of the Complementary and Alternative Medicine team who participated in study design and data collection: Mr. Kenichi Yamada, Mr. Hiroyuki Kikuchi, Ms. Miyuki Ohmura, and Ms. Maya Okumura in Sendai ART clinic.

\section{Authors' contributions}

$\mathrm{KO}$ and $\mathrm{HY}$ organized the study. MK, RM and AS collected and analyzed the data. $\mathrm{KO}$ drafted the manuscript. $\Pi \mathrm{T}$ and $\mathrm{HM}$ revised the manuscript. All authors have read and approved the manuscript.

\section{Authors' information}

Not applicable.

\section{Funding}

No external funding sources were used for this study.

\section{Availability of data and materials}

The datasets during and/or analyzed during the current study available from the corresponding author on reasonable request.

Ethics approval and consent to participate

Not applicable.

\section{Consent for publication}

Written informed consent was obtained from the patient for publication of this case report and accompanying images.

\section{Competing interests}

The authors declare that they have no competing interests.

\section{Author details}

${ }^{1}$ Fukushima Medical Center for Children and Women, Fukushima Medical University, 1 Hikarigaoka, Fukushima City, Fukushima 960-1295, Japan. ${ }^{2}$ Sendai ART Clinic, 206-13 Nagakecho, Miyagino, Sendai, Myagi 983-0864, Japan. ${ }^{3}$ Department of obstetrics and gynecology, Showa University, 1-5-8 Hatanodai, Shinagawa, Tokyo 142-8555, Japan.

Received: 27 September 2019 Accepted: 17 January 2020

Published online: 29 January 2020

\section{References}

1. Papanikolaou EG, Fatemi H, Venetis C, Donoso P, Kolibianakis E, Tournaye $H_{\text {, }}$ Tarlatzis B, Devroey P. Monozygotic twinning is not increased after single blastocyst transfer compared with single cleavage-stage embryo transfer. Fertil Steril. 2010;93(2):592-7.

2. Osianlis T, Rombauts L, Gabbe M, Motteram C, Vollenhoven V. Incidence and zygosity of twin births following transfers using a single fresh or frozen embryo. Hum Reprod. 2014;29(7):1438-43.

3. Vega M, Zaghi S, Buyuk E, Jindal S. Not all twins are monozygotic after elective single embryo transfer: analysis of 32,600 elective single embryo transfer cycles as reported to the Society for Assisted Reproductive Technology. Fertil Steril. 2018;109(1):118-22.

4. MacGillivray I. Epidemiology of twin pregnancy. Semin Perinatol. 1986;10(1):4-8.

5. Nakasuji $T$, Saito $H$, Araki $R$, Nakaza A, Nakashima A, Kuwahara A, Ishihara O, Irahara M, Kubota T, Yoshimura Y, et al. The incidence of monozygotic twinning in assisted reproductive technology: analysis based on results from the 2010 Japanese ART national registry. J Assist Reprod Genet. 2014;31(7):803-7.

6. Kawachiya S, Bodri D, Shimada N, Kato K, Takehara Y, Kato O. Blastocyst culture is associated with an elevated incidence of monozygotic twinning after single embryo transfer. Fertil Steril. 2011:95(6):2140-2.

7. Derom C, Vlietinck R, Derom R, Van den Berghe H, Thiery M. Increased monozygotic twinning rate after ovulation induction. Lancet. 1987;1 (8544): 1236-8. 
8. Steinman G, Valderrama E. Mechanisms of twinning. III. Placentation, calcium reduction and modified compaction. J Reprod Med. 2001;46(11):995-1002.

9. Menezo YJ, Sakkas D. Monozygotic twinning: is it related to apoptosis in the embryo? Hum Reprod. 2002;17(1):247-8.

10. Wei Z, Cao Y, Cong L, Zhou P, Zhang Z, Li J. Effect of metformin pretreatment on pregnancy outcome of in vitro matured oocytes retrieved from women with polycystic ovary syndrome. Fertil Steril. 2008;90(4):1149-54.

11. Trounson A, Wood C, Kausche A. In vitro maturation and the fertilization and developmental competence of oocytes recovered from untreated polycystic ovarian patients. Fertil Steril. 1994;62(2):353-62.

12. Demeestere I, Simon P, Emiliani S, Delbaere A, Englert Y. Orthotopic and heterotopic ovarian tissue transplantation. Hum Reprod Update. 2009;15(6): 649-65.

13. Cao $Y X$, Chian RC. Fertility preservation with immature and in vitro matured oocytes. Semin Reprod Med. 2009;27(6):456-64.

14. Lindenberg S. New approach in patients with polycystic ovaries, lessons for everyone. Fertil Steril. 2013;99(5):1170-2.

15. Kanaya $H$, Murata $Y$, Oku H, Hashimoto $S$, Morimoto $Y$, Murata T, Sugihara $K$, Nagata F, Nakaoka Y, Fukuda A. Successful monozygotic twin delivery following in vitro maturation of oocytes retrieved from a woman with polycystic ovary syndrome: case report. Hum Reprod. 2006;21(7):1777-80.

16. Hashimoto S, Murata Y, Kikkawa M, Sonoda M, Oku H, Murata T, Sugihara K, Nagata F, Nakaoka Y, Fukuda A, et al. Successful delivery after the transfer of twice-vitrified embryos derived from in vitro matured oocytes: a case report. Hum Reprod. 2006;22(1):221-3.

17. Revised 2003 Consensus on diagnostic criteria and long-term health risks related to polycystic ovary syndrome (PCOS). Hum Reprod. 2004;19(1):41-7.

18. Hall JG. Twinning. Lancet. 2003;362(9385):735-43.

19. Tocino A, Blasco V, Prados N, Vargas MJ, Requena A, Pellicer A, Fernandez-Sanchez M. Monozygotic twinning after assisted reproductive technologies: a case report of asymmetric development and incidence during 19 years in an international group of in vitro fertilization clinics. Fertil Steril. 2015;103(5):1185-9.

20. Shibuya Y, Kyono K. A successful birth of healthy monozygotic dichorionic diamniotic (DD) twins of the same gender following a single vitrifiedwarmed blastocyst transfer. J Assist Reprod Genet. 2012;29(3):255-7.

21. Ikemoto Y, Kuroda K, Ochiai A, Yamashita S, Ikuma S, Nojiri S, Itakura A, Takeda S. Prevalence and risk factors of zygotic splitting after 937848 single embryo transfer cycles. Hum Reprod. 2018;33(11):1984-91.

22. Fadini R, Mignini Renzini M, Guarnieri T, Dal Canto M, De Ponti E, Sutcliffe A, Shevlin M, Comi R, Coticchio G. Comparison of the obstetric and perinatal outcomes of children conceived from in vitro or in vivo matured oocytes in in vitro maturation treatments with births from conventional ICSI cycles. Hum Reprod. 2012;27(12):3601-8.

23. Buckett WM, Chian RC, Holzer H, Dean N, Usher R, Tan SL. Obstetric outcomes and congenital abnormalities after in vitro maturation, in vitro fertilization, and intracytoplasmic sperm injection. Obstet Gynecol. 2007;110(4):885-91.

24. Saravelos SH, Zhang T, Chung JP, Sun LM, Sun Y, Li TC, Chen ZJ. Monochorionic quadramniotic and triamniotic pregnancies following single embryo transfers: two case reports and a review of the literature. J Assist Reprod Genet. 2016;33(1):27-32.

25. Alikani M, Noyes N, Cohen J, Rosenwaks Z. Monozygotic twinning in the human is associated with the zona pellucida architecture. Hum Reprod. 1994;9(7):1318-21.

26. da Costa AA, Abdelmassih S, de Oliveira FG, Abdelmassih V, Abdelmassih R, Nagy ZP, Balmaceda JP. Monozygotic twins and transfer at the blastocyst stage after ICSI. Hum Reprod. 2001;16(2):333-6.

27. Skiadas CC, Missmer SA, Benson CB, Gee RE, Racowsky C. Risk factors associated with pregnancies containing a monochorionic pair following assisted reproductive technologies. Hum Reprod. 2008;23(6):1366-71.

28. Luke B, Brown MB, Wantman E, Stern JE. Factors associated with monozygosity in assisted reproductive technology pregnancies and the risk of recurrence using linked cycles. Fertil Steril. 2014;101(3):683-9.

29. Siristatidis CS, Maheshwari A, Vaidakis D, Bhattacharya S. In vitro maturation in subfertile women with polycystic ovarian syndrome undergoing assisted reproduction. Cochrane Database Syst Rev. 2018;11:CD006606.

\section{Publisher's Note}

Springer Nature remains neutral with regard to jurisdictional claims in published maps and institutional affiliations.

\section{Ready to submit your research? Choose BMC and benefit from:}

- fast, convenient online submission

- thorough peer review by experienced researchers in your field

- rapid publication on acceptance

- support for research data, including large and complex data types

- gold Open Access which fosters wider collaboration and increased citations

- maximum visibility for your research: over $100 \mathrm{M}$ website views per year

At BMC, research is always in progress.

Learn more biomedcentral.com/submissions 